\title{
Pulmonary benign metastasizing leiomyoma simultaneously diagnosed with uterine leiomyoma at first visit before hysteromyomectomy
}

\author{
Yue Wü,^, Liwen Fan", Yuxu Niu, Yun Wu, Wen Gao \\ Department of Thoracic Surgery, Huadong Hospital Affiliated to Fudan University, Shanghai, China \\ \#These authors contributed equally to this work. \\ Correspondence to: Yun Wu; Wen Gao. Department of Thoracic Surgery, Huadong Hospital Affiliated to Fudan University, No. 221 West Yanan \\ Road, Shanghai 200040, China. Email: a.oret@live.cn; gaowenchest@163.com.
}

\begin{abstract}
Pulmonary benign metastasizing leiomyoma (PBML) represents a rare disease which is mostly diagnosed in reproductive-age women with a hysteromyomectomy history. This disease could occur in any age group, but more common in women of late fertility. And its clinical processes are closely linked to sex hormone levels. Here we describe a case of PBML simultaneously diagnosed with uterine leiomyoma at patient's first visit without a hysteromyomectomy history. The patient was a 37-year-old woman presenting with recurrent lower abdominal discomfort for more than half a year. No nausea, cough, dyspnea, hemoptysis, or abdominal distension was found. Additionally, the menstruation of the patient was regular. Image examinations confirmed a single solid pulmonary nodule in the right upper lobe, multiple fibroids of the uterus, and left adnexal nodules at her first visit. Laparoscopic hysteromyomectomy and pulmonary segmentectomy were sequentially performed with an interval of one month. Pathological findings were consistent with benign metastasizing leiomyomas. Estrogen and progesterone receptors were positive by immunohistostaining of the tumors. In conclusion, when confronted with female patients, who are at childbearing age with or without a history of uterine fibroids surgery, with single or multiple lesions in the lung that highly resemble metastatic tumors, but fail to find the primary malignant tumor clinically, the possibility of PBML must be considered. And sequential surgical treatment could be arranged.
\end{abstract}

Keywords: Pulmonary benign metastasizing leiomyoma (PBML); uterine leiomyoma; sequential pulmonary segmentectomy; case report

Submitted May 06, 2020. Accepted for publication Nov 16, 2020.

doi: $10.21037 /$ tcr-20-2016

View this article at: http://dx.doi.org/10.21037/tcr-20-2016

\section{Introduction}

Pulmonary benign metastasizing leiomyoma (PBML) is a rare disease that represents the most likely extrauterine metastasis of uterine leiomyoma (1). The first case of PBML was discovered and reported by Steiner in 1939 (2). This disease could occur in any age group, but more common in women of late fertility. And its clinical processes are closely linked to sex hormone levels (3). Additionally, most cases were discovered through chest radiographs or chest computed tomography (CT) during routine medical examinations without clinical symptom, while a small number of patients had symptoms of cough, dyspnea, or chest pain. However, it was difficult to diagnose PBML only with imaging and laboratory diagnosis, which was often misdiagnosed as pneumonia, bronchitis, tuberculosis, or even metastatic lung cancer. Surgical lung biopsy is the gold standard for diagnosis (2). Interestingly, most

^ ORCID: 0000-0001-5101-6035. 

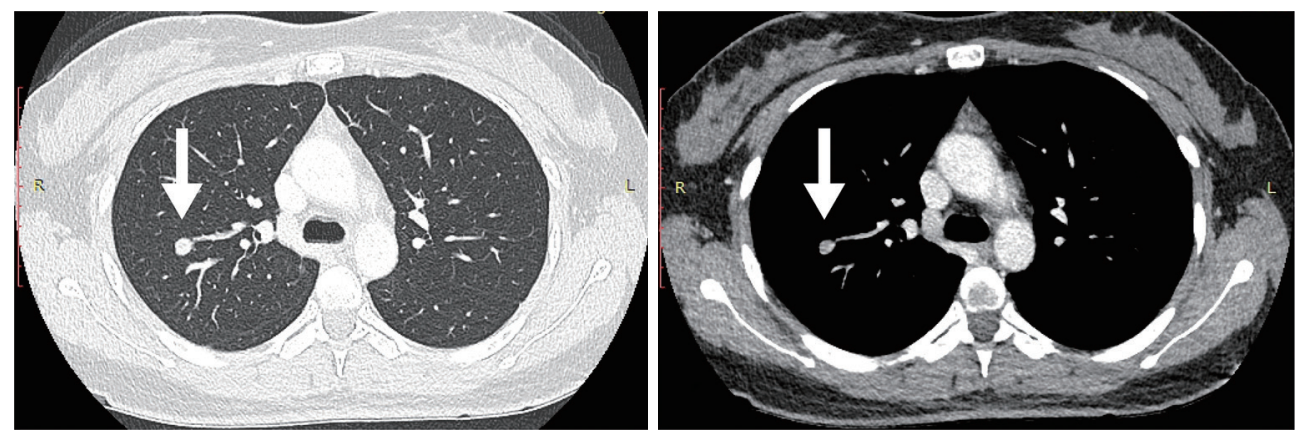

Figure 1 Computed tomography of a solid pulmonary nodule of the right upper lobe in the patient with benign metastasizing leiomyoma (white arrow).

PBML cases had a history of hysteromyomectomy, and the majority of them were diagnosed within 15 years after hysteromyomectomy (3). Here we describe a case of PBML simultaneously diagnosed with uterine leiomyoma at first visit without a hysteromyomectomy history. We present the following case in accordance with the CARE reporting checklist (available at http://dx.doi.org/10.21037/tcr-202016).

\section{Case presentation}

A 37-year-old woman presented with recurrent lower abdominal discomfort for more than half a year. No nausea, cough, dyspnea, hemoptysis, or abdominal distension was found. Additionally, the menstruation of the patient was regular. There were no abnormalities in routine laboratory examinations, tumor markers, and pulmonary function tests. She had no medical, family, and psycho-social history of relative diseases. This was her first visit to this issue, and she had no relevant past interventions. All procedures performed in studies involving human participants were in accordance with the ethical standards of the institutional research committee and the Helsinki Declaration (as revised in 2013). Written informed consent was obtained from the patient.

A contrast-enhanced thoracic CT scan revealed a $9 \mathrm{~mm} \times 7 \mathrm{~mm}$ solid nodule in the right upper lobe with shallow lobules at the margin, clear boundary, and obvious enhancement; no enlarged lymph nodes in the mediastinum and hila. And two radiologists considered it to be benign or low-grade malignant (Figure 1). A gynecological ultrasound indicated multiple fibroids in the uterus and left adnexal nodules.

Laparoscopic hysteromyomectomy and left adnexectomy were performed on September 17, 2019. The gross examination showed the surface cut of the tumor was pale and tough, and its tissue had a whirlpool shape. The postoperative pathology indicated uterine leiomyoma (Figure $2 A, B)$. The results of immunohistochemistry were as follows: Ki-67 (1\%,+), ER (+), PR (+), SMA (+), Des (+), CD117 (-), CD34 (-), DOG1 (-), and B-Catenin (-). After postoperative rehabilitation and follow-up examinations, the patient received apical and posterior segmentectomy (the location of the nodule was too deep to perform wedge resection) by video-assisted thoracic surgery on October 21,2019 . The intraoperative frozen section demonstrated a spindle cell tumor. The gross examination showed a pale 0.8 -cm-diameter nodule with a clear boundary with normal lung tissue. Postoperative pathological findings suggested a leiomyoma (Figure $2 C, D$ ). And immunohistochemical analysis revealed a result of SMA (+), Des (+), ER (+), PR (+), Ki-67 (5\%, +), CK7 (-), CD34 (-), STST6 (-), ALK (-), CD68 (-). Therefore, this case was diagnosed as benign metastasizing leiomyoma. Afterward, the patient received a treatment of tamoxifen. No radiological evidence of progressive disease and further distant metastasis were found in a twelve-week follow-up. The patient had no relevant symptoms and the prognosis was good.

\section{Discussion}

PBML is a rare benign tumor from the uterus, which usually occurs in women of childbearing age (3). The growth of this type of tumor mainly depends on the level of estrogen and progesterone. Tumor progression is stimulated by estrogen, and tumor regression is stimulated by progesterone. The tumor could regress on its own after pregnancy or menstruation stop (4). 

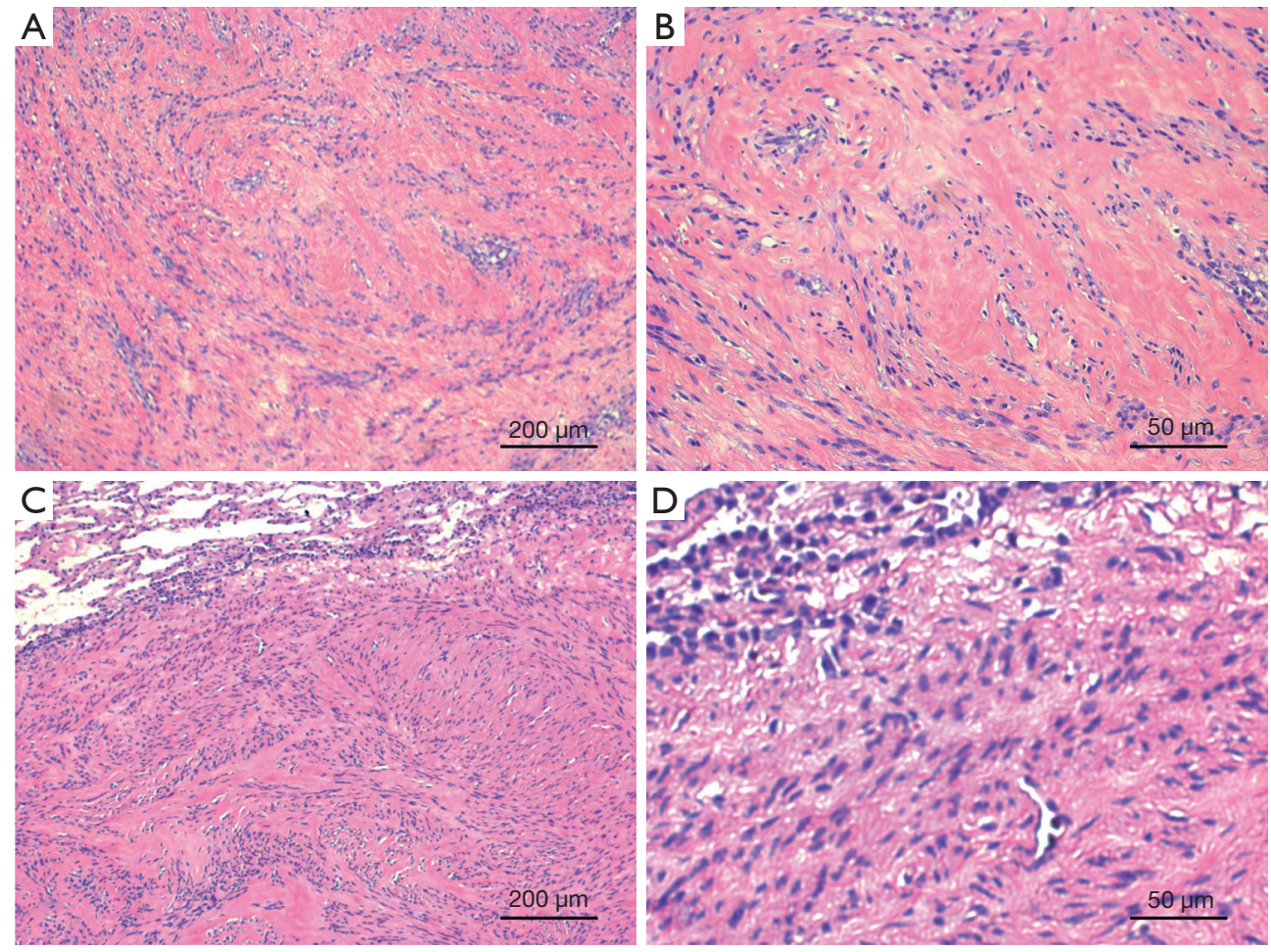

Figure 2 Hematoxylin-eosin staining showed (A: 100x, B: 400x) the uterine tumor cells were spindle like and arranged in a whirlpool shape, (C: $100 \times$, D: $400 \times$ ) fusiform smooth muscle cells aggregated and arranged in a palisade form in the pulmonary tumor, and no atypia or malignant cells existed.

Most PBML cases had a history of hysteromyomectomy, and the majority of them were diagnosed within 15 years after hysteromyomectomy (3). However, the pathogenesis of this disease is still vague (3). The widely accepted theory is hematogenous dissemination. The metastatic mechanism may be that uterine surgery (including curettage, hysteromyomectomy, hysterectomy, etc.) increases the possibility of surgically induced hematogenous spread (5). Nevertheless, this theory cannot explain this case. In this case, the pulmonary nodule had appeared before uterine surgery. And similar cases are extremely rare. Therefore, the pathogenesis of PBML still needs further research.

Imaging examination is the main method to diagnose PBML, which has been reported in the literature as solid lung nodules. They are mostly round or quasi-circular with uniform density, no calcification, clear and smooth borders, no burrs, and lobules and cavities or not. Nodules could increase in number and enlarge in size during follow-up, but the growth rate is slow (6). Horstmann retrospectively reviewed the imaging findings of 23 PBML patients and found that bilateral multiple solid pulmonary nodules were the most common type (16 cases, $70 \%$ ), of which a few had lobules or cavities; followed by unilateral multiple masses ( 4 cases, $17 \%$ ), and single mass was the least (3 cases, 13\%). Diffuse miliary lung lesions and tracheobronchial stenosis or obliteration were rare according to the literature. Additionally, the bronchial epithelium, pleura, and mediastinal lymph nodes were generally not involved by PBML (7).

In this case, the CT manifestation of the lesion was a small single solid nodule in the right upper lobe, with shallow lobes at the margin. By imaging alone, it is not easy to distinguish PBML from a lung metastasis of malignant tumors. When confronted with female patients, who are at childbearing age with a history of uterine fibroids surgery, with single or multiple lesions in the lung that highly resemble metastatic tumors, and fail to find the primary malignant tumor clinically, the possibility of PBML must be considered. The diagnosis of PBML depends on aspiration biopsy or postoperative pathological examination as well as immunohistochemistry (2).

As for the treatment, we followed the principle of treating 
tumors. First, we removed the uterine fibroids (which can be regarded as the main lesion), and then removed the lung metastasis (PBML). sequential resection reduced the trauma to the patient compared with the synchronous surgical treatment of all lesions. Both operations were minimally invasive surgery to reduce surgical trauma. hormonal manipulation after surgery was intended to consolidate the outcome. The patient reported good recovery after the operation and no obvious discomfort. However, we did not innovate treatment methods and still were guided by experience.

Like uterine leiomyoma, PBML is also a hormonedependent lesion with positive expression of estrogen receptor and progesterone receptor by immunohistochemical examination. Besides, many studies reported that hormonal therapy was effective $(8,9)$. However, surgical treatment should be preferred for resectable cases. And all patients should have long-term follow-up for changes in sex hormone levels and lung conditions postoperatively.

\section{Acknowledgments}

The authors wish to thank all of the staff at the Department of Thoracic Surgery, Huadong Hospital Affiliated to Fudan University.

Funding: The authors appreciate the grant from the Young Scientists Fund of the National Natural Science Foundation of China (No. 81702252).

\section{Footnote}

Reporting Checklist: The authors have completed the CARE reporting checklist. Available at http://dx.doi.org/10.21037/ tcr-20-2016

Conflicts of Interest: All authors have completed the ICMJE uniform disclosure form (available at http://dx.doi. org/10.21037/tcr-20-2016). The authors have no conflicts of interest to declare.

Ethical Statement: The authors are accountable for all aspects of the work in ensuring that questions related to the accuracy or integrity of any part of the work are appropriately investigated and resolved. All procedures performed in studies involving human participants were in accordance with the ethical standards of the institutional research committee and the Helsinki Declaration (as revised in 2013). Written informed consent was obtained from the patient.
Open Access Statement: This is an Open Access article distributed in accordance with the Creative Commons Attribution-NonCommercial-NoDerivs 4.0 International License (CC BY-NC-ND 4.0), which permits the noncommercial replication and distribution of the article with the strict proviso that no changes or edits are made and the original work is properly cited (including links to both the formal publication through the relevant DOI and the license). See: https://creativecommons.org/licenses/by-nc-nd/4.0/.

\section{References}

1. Sawai Y, Shimizu T, Yamanaka Y, et al. Benign metastasizing leiomyoma and 18-FDG-PET/CT: A case report and literature review. Oncol Lett 2017;14:3641-6.

2. Steiner PE. Metastasizing fibroleiomyoma of the uterus: Report of a case and review of the literature. Am J Pathol 1939;15:89-110.7.

3. Kayser K, Zink S, Schneider T, et al. Benign metastasizing leiomyoma of the uterus: documentation of clinical, immunohistochemical and lectin-histochemical data of ten cases. Virchows Arch 2000;437:284-92.

4. Miller J, Shoni M, Siegert C, et al. Benign Metastasizing Leiomyomas to the Lungs: An Institutional Case Series and a Review of the Recent Literature. Ann Thorac Surg 2016;101:253-8.

5. Egberts JH, Schafmayer C, Bauerschlag DO, et al. Benign abdominal and pulmonary metastasizing leiomyoma of the uterus. Arch Gynecol Obstet 2006;274:319-22.

6. Allen MS. Multiple benign lung tumors. Semin Thorac Cardiovasc Surg 2003;15:310-4.

7. Horstmann JP, Pietra GG, Harman JA, et al. Spontaneous regression of pulmonary leiomyomas during pregnancy. Cancer 1977;39:314-21.

8. Jo HC, Baek JC. Case of pulmonary benign metastasizing leiomyoma from synchronous uterine leiomyoma in a postmenopausal woman. Gynecol Oncol Rep 2018;26:33-6.

9. Aka N, Iscan R, Köse G, et al. Benign Pulmonary Metastasizing Leiomyoma of the Uterus. J Clin Diagn Res 2016;10:QD01-3.

Cite this article as: $\mathrm{Wu} \mathrm{Y,} \mathrm{Fan} \mathrm{L,} \mathrm{Niu} \mathrm{Y,} \mathrm{Wu} \mathrm{Y,} \mathrm{Gao} \mathrm{W.}$ Pulmonary benign metastasizing leiomyoma simultaneously diagnosed with uterine leiomyoma at first visit before hysteromyomectomy. Transl Cancer Res 2021;10(1):567-570. doi: 10.21037/tcr-20-2016 